\title{
Analysis the Traces Identified in the Case of Violent Crimes
}

\author{
Ovidiu Andrei Hamburda \\ Faculty of "Economic Sciences and Public Administration," University “Stefan cel Mare," Suceava, Romania \\ Master in Criminal Sciences at "Dimitrie Cantemir" University, Bucharest, Romania \\ ovidiu.andrei20@yahoo.com
}

\begin{abstract}
The studies and research show that in almost all countries of the world, violent crimes are on the rise. Against this background, the criminal prosecution authorities - the police and the prosecutor's office, as institutions of the rule of law, improve investigative procedures, using the scientific means and methods necessary to prove guilty and to prosecute the perpetrators. Human-environment interaction gives rise to the most different traces that can be useful in determining the time or succession of occurrence events, as well as determining human behavior in this process. Any human activity deployed in time and space, through physical movements, leaves in that place different changes from the previous situation, which is preserved for an appreciable period of time. Depending on all the elements presented, you can reconstitute the dynamic picture of the deed. The traces come to occupy the central place in science of forensics, so that at present no one can imagine a crime committed by physical movements of the person, without the proper traces being made at the place where it was committed.
\end{abstract}

KEYWORDS: crime, forensics, investigation, movement, police, traces

\section{Introduction}

The trace is a change created at the scene of the crime and in the process of committing the crime, by physical movements at that time, by those involved in criminal activity, useful to forensic research. The first condition highlights the need for a causal relationship between the offense and the change made. The second condition is limited to the need for the modification produced to be useful for forensic research. According to the Code of Criminal Procedure of Romania, in article 197 there are objects as material evidence that contain a trace of the committed deed, as well as any other objects that can serve to find the truth are material means of proof. According to the same article, criminal assets are used as evidence which have been or have been used to commit an offense, as well as objects that are the product of the offense (Code of Criminal Procedure of Romania).

According to their training, traces are classified into: reproductive traces: static (trace created by a car tire) and dynamic (a trace created by the ax in the wood mass).

By the degree of plasticity of the receiving object:

- deep foot step: traces in clayey ground, in the snow;

- surface traces: stratification: mud on shoes, sweat and destratification: traces created on a dusty surface;

By the nature of the creative object:

- traces of hands and feet; traces of lips, teeth, created by clothing;

- biological traces (Asanache and Dragomir 1976).

\section{Traces of hands}

Traces of hands are created as a result of human activity, being produced by hand contact with various environmental objects by supporting, gripping, pushing, etc. Of these, the human body is created directly, those of the hands were and are also used when identifying the person to a much greater extent than any other type of trace. This is due to the numerous lines running through the faces of the fingers and fingers. The traces found at the scene are called "finger traces" and the ones that create experimental are "digital impressions", papillary relief photographs (traces or impressions) are called "dactylograms".

Due to papillary lines, traces of hands are formed when the objects in the environment touch with the flanks or the surface of the palm. On objects with dry surfaces are created when hand and 
object received are relatively clean. This is explained by the existence on the palm and the breaking of a secreted substance of the skin, the substance made up of organic and inorganic compounds, which does not evaporate with the passage of time. Traces on the surface of the inviting object is kept, with the details created, a relatively long time.

The issues that can be solved by the digital tracing and palm tracing expertise are as follows: identification of the person who left the next incrimination, - identification of the unknown body after the papillary drawings, - verification of the fingerprint signatures, - identification of the person from whom two or more many dactyloscopic files written on different names, the establishment of the route formation mechanism, the delineation of partially overlapped paths. Dactiloscopy is "science on the study of papillary drawings" (Constantin Turai). By means of digital traces, if they are pretty clear, with sufficient detail, it is safe to identify the person who created them. Achieving this performance in criminality research can be achieved by the anatomical relief on the front of the palm, and especially by a flank, which is in the form of ridges and fingers. The ridges of this relief, in the form of a parallel crest, are called papillary lines, and the recesses, interpapillary wrinkles, and their whole ensemble is called papillary relief or papillary drawing.

The visible traces are left by fingers dirty with coloring substances such as blood, paint, ink, mud, dust, cement, soot, etc. Fingers dirty with blood or paint and repeatedly applied on different objects will leave the fingerprints in the form of dents, and then the creases of the crests will be white and those of the colored hides. In this case, the hand marks will appear as a negative of the papillary drawings.

Papillary reflex properties (Beliș et al. 1995):

- Longevity: The papillary relief forms before birth, it is completed by the sixth month of the fetus and continues until the skin breaks down in the process of rotting.

- Fixity involves preserving the original features without change in the course of existence, with the exception of growth.

- Uniqueness: Every person has his or her own, unrepeatable of the other person.

- Inalterability: Lifetime can not be wiped out by physical or chemical means if the dermis layer is not destroyed, called papillary relief or papillary drawing.

\section{Traces of feets}

Traces of feets are an important category of footprints inevitably created at the scene. However, they are found or used in rarer realistic identification research, believed to have fewer possibilities for individualization, due to the relatively small number of characteristic elements, except those specific to the papillary ridges on the sole of the foot. First of all, many times, in the case of such traces, the papillary relief is either very bent or when the trace has been stuffed with foreign substances, so in both situations there is little of its details. Secondly, very often that object does not have a surface smooth enough to receive and retain the papillary relief detail. Traces of foot are easily discovered at the scene. Fixing can be accomplished by several procedures: by describing in minutes, shooting, molding (deep), copying with the adhesive film (surface ones).

- The description refers to: the area, the nature of the receiving object, its color, dimensions (heel, sole, length, width, height);

- Taking a picture involves a photo of the main objects with the device on the tripod, adequate illumination; detail of photo with perpendicular lens, scaled photo;

- Mounting by molding is performed after the first two processes; it strengthens the trace if it is formed in snow or sand, then pour the molding paste, usually from dental gypsum;

- Copying with the adhesive film is done after the handwriting process.

Tracking - consists of a series of several consecutive traces created by both feet on the trail.

Footprints created by or with the foot are as follows (Palcu 2006):

- The traces of the foot plant, or the foot formed by the foot, are the most valuable for individualization, as the plant print with its own papillary characteristics as well as the morphophysiological particle can serve to a certain identification of the individual, equivalent to 
fingerprint identification. Planetary impressions, or even better, the plantar papillary design can have fingerprint, the same importance as digital or palm impressions.

- Footsteps of leg or stockings reproduce the general shape of the foot plant, its regions and fabric. They can serve for group determination and even identification.

- Traces of footwear, if properly formed (such as static, deep, soft ground), may reflect characteristic features that are useful in identifying, although they have fewer individual elements.

\section{Traces of teeth and lips}

Human teeth, in their general appearance of the species, have many general and individual characteristics that can be observed in any person either in his or her usual speech or in the traces created by biting. In the process of speech, the person discovers his front teeth, incisors and canines, and thus can be identified as criminalistic within the portrait spoken. By biting leaves on the welcoming objects the characteristics of incisors and canines, after which it can be identified. The individual features of the incisive and canine teeth of the human being that distinguish them from one individual to another are: varied width, the different distances between them, the difference in position on the two arches, the degree of wear, some peculiarities created by medical treatments (The Criminal Law Magazine 2011).

To these traces, created on the human body, one has to bear in mind that after the bite production, the skin relaxes, causing changes in the created traces in terms of the width of the teeth and the distances between them. However, through the traces of teeth printed on the human body, it is possible to arrive at definite conclusions about suspects.

The tooth trail offers, from the scene, the first information about the constitutional type and the age of the person who created it (Mircea 2001).

It has long been found that human lips have partculiarities through their coral wrinkles. It is established with certainty that the coral lines of the lips have various individual characteristics with an appreciable duration of existence in terms of the shapes and positions they have in the whole of the labial relief. These lines, by directly contacting certain objects in the surrounding world, leave traces on the objects, after which they can be reached until the labial relief is identified. The lip traces, in most cases, are latent. For this reason, the judiciary has to look very carefully at the objects of the crime scene and, after discovering them, proceed to highlight them. Always when the traces of the lips are visible, they are easily discovered by simply examining the suspect objects with the naked eye. However, very rarely visible lips are created, only when lips are lipsticked.

\section{Traces of blood}

The presence of blood traces at the scene of the crime in all cases implies the injury of the victim's body integrity. In general, such types of traces are found in the case of murder, robbery, rape, body injury, attempted murder, etc. Interpretation of blood has proven to be extremely vital in cases where the cause of death was questionable, and the determination of whether it was murder, suicide, accident or natural death was necessary for the criminal process. Being a fluid mixture made up of cellular and plasma components, blood, when it comes from the body into the external environment, as a result of the traumas produced by various forces, it naturally obeys the laws of physics.

The form of blood traces encountered at the site of an offense is mainly influenced by the amount of blood that has emerged from the human body, the speed at which it moves to the receiving surface, and the angle at which it meets the surface. Referring to these key factors, there are several types of blood traces, such as (Moraru 1997):

- Traces formed by contact or impact traces with different speeds;

- Traces formed by the free fall of blood droplets on horizontal surfaces;

- Traces formed by splashing, splashing, throwing or designing blood.

Traces formed by contact include: scratches and prints made as a result of the dynamic contact between the blood supply and the various areas of the crime scene. These may occur as a result of static contact between parts of the aggressor's body or previously contaminated objects. 


\section{Traces of sperm, saliva and hairs identification}

Semen traces consist of seminal fluid resulting from the secretion of male seminal glands during sexual intercourse or sexual perversion, masturbation, nocturnal pollution or ejaculation due to pathological conditions. They are formed by depositing the sperm removed on different supports in the immediate vicinity of the place where ejaculation occurs, such as intimate linen, sheets, clothing, carpets, body of the victim or the offender, especially on the thighs, abdomen, pubescent brushes, hands, breasts, breasts, anus, vagina (Ifrim and Niculescu 1988).

Their forensic value is of particular importance in investigating offenses related to sexual life, certain murder crimes in suicide cases of men by hanging. The forms and aspects of the presentation of sperm traces depend in most cases on the nature of the support, the way the semen has come into contact with the support, the length of time elapsed between training and the time of discovery, the diseases suffers the individual, and if left by several men, it also influences to a great extent this factor. For the success of this kind of expertise, we recall the need to comply with the rules of lifting and shipping urgently to the laboratory of biological stains under conditions that do not allow their alteration, such as the packaging of wet objects bearing biological traces in plastic bags or bags. It is very important that caution should be exercised when harvesting and packaging the samples (not only biological ones), preventing confusion by reversing them. Expertise to determine the genetic profile based on seminal traces, as well as blood or saliva, is one of the safest means of investigation.

\section{Grafoscoptic expertise}

It is ordered in cases where, at the scene of the crime or at the victim's home, documents are revealed from which the data may be revealed regarding the deed or even the person of the author, typical of which is letters of threat. The graphological expertise is also indispensable in the case of the abduction of persons, when it is necessary to establish the authenticity of the victim's writing, which can even be added to the decipherment of some of his messages, concealed in the text.

\section{Ballistic expertise}

The forensic expertise of the main traces of the shooting consists of examining the inlets and outlets of the channels formed both on the human body and on the objects with which the bullet has come into contact. They are also subject to scrutiny and riches.

Ballistic expertise is aimed at two directions:

- Identifying or determining the category of the weapon that was fired, following the trail left on the tube and on the bullet (Stancu 2010);

- Studying the main and secondary traces of the victim's clothing in order to determine the direction and distance from which it was fired.

Regarding the expertise of the entrance openings, a first problem to solve is whether to determine whether or not these holes are the consequence of using a firearm or a weapon of another nature. The answer can be based on the study of the general characteristics reflected by the $\log$ entry, characteristics that differ depending on the nature of the object being shot (tissue, wood, metal, etc.).

Along with these features of the orifice are also considered the individual characteristics specific to the secondary factors of the shot, such as: traces of soot, burns, tattoos and gas breaks, as well as friction and metallization rings formed regardless of the distance from which it was fired. All these aspects are classified on the basis of the comparative examination between the traces in question and the traces of the experiments carried out with weapons suspected to have been used on supports similar to those found on the spot (Goșa 2009).

By its complexity, ballistic expertise requires the same close co-operation between the forensic expert and the forensic doctor. 


\section{Conclusions}

The tracking activity on the spot has a particularly important role to play in clarifying all the circumstances in which the offense under the criminal law has been committed and the forensic interpretation by the specialists using research methods: logical reasoning, observation, description , experiment, analysis, measurements, etc. can be a guarantee of transformation of each trace into the sample and the formation of a solid probative system, which will support a fair solution that will be pronounced in that case.

\section{References}

Asanache, Gh. And Dragomir, M. 1976. Practically Treated by Forensics, vol. II. Bucharest: The Ministry of Interior Publishing House.

Beliș, V., et al. 1995. Treaty of legal medicine. Bucharest, Romania: Teora Publishing House.

Code of Criminal Procedure of Romania.

Goşa, D. 2009. Forensics - University course, vol. I, Sibiu, Romania: Alma Mater Publishing House.

Rasfoiesc.com. 2019. "Footsteps of Hands." Available at http://www.rasfoiesc.com/legal/criminalistica/URMELE-DEMAINI48.php.

Ifrim. M., and Niculescu, Gh. 1988. Anatomy compedium, Bucharest: Didactic and Pedagogical Publishing House. Ion, Mircea. 2001. Criminalistica, Bucharest: Lumina LEX Publishing House.

Moraru, I. 1967. Medicină Legală, Bucharest: Romania: Medical Publishing House.

Palcu, P. 2006. The tactic of forensic research on the spot, "Vasile Goldiş." Arad, Romania.

Romanian Criminal Code.

Stancu, E. 2010. Treaty of forensics, vol. V. Bucharest: Universul Juridic Publishing House.

The criminal law magazine. 2011. Bucharest: Universul Juridic Publishing House. 